\title{
Decolonizing ecology and evolution is a long road
}

\author{
An update on our progress in anti-racist actions and confronting colonial science.
}

$\mathrm{L}$ ast year, we wrote two editorials (in June and September) calling for anti-racist action in the ecology and evolution community and committing to that action as a journal. We noted that we should not let the issue drop down the priority list, and that we should report on it regularly.

Two Perspective articles in this issue address the related issues of anti-racism and decoloniality. Cronin et al. begin their article by describing the history of racism in ecology and evolution, including racism in species nomenclature, scientific racism, and colonial practices in fieldwork and conservation. They then present a toolkit with which to deconstruct this legacy and foster anti-racism in the future. The toolkit includes interventions in classrooms by teaching about colonialism; in research groups by promoting an inclusive culture; and in institutional departments by establishing policies for hiring, tenure and retention.

In one of our editorials last year, we noted that most of the content on amplifying diverse voices we had published at that point came from North American authors, and that we wanted to publish a more global perspective, particularly from authors in the Global South. The second Perspective in this issue, by Trisos and colleagues, presents a framework for a more globally inclusive and ethical ecology, which confronts colonial histories and respects a diverse range of contributors and types of knowledge. The recommendations set out in these two Perspectives provide the basis for researchers everywhere to make meaningful changes to combat inequality.

Several other recent articles have confronted the Global North bias that is prevalent across science. In a Comment article, Dolors Armenteras sets out guidelines for ensuring that collaborations between researchers from higher- and lower-income countries are not exploitative. These include working together for the long term and with real equality, and avoiding what is known as parachute research. This practice, in which researchers from wealthy institutions carry out fieldwork or institutional visits in lower-income countries and take back data or resources without meaningful collaboration with local researchers, is particularly problematic in palaeontology. It was the subject of another recent editorial in the context of Burmese amber, and has led us to make updates to Nature Portfolio editorial policies.

Another recent Comment article, from Wehi et al., advocates for an Indigenous Māori approach to management and conservation of the Antarctic, which, despite an ostensibly collaborative framework, has been dominated by colonial viewpoints. Similarly, Dyhia Belhabib argues in a World View article that we need to move away from a narrative on ocean conservation that is dominated by the concerns and prejudices of scientists and citizens from the Global North.

Ethical engagement with Indigenous communities is also the topic of a Comment by Handsley-Davis and colleagues, here in the context of environmental DNA. One issue they discuss is the potential implications of environmental DNA studies for land claims, where findings taken out of context could be used to counter such claims. Land acknowledgements (also known as 'acknowledgements of country', referring to statements recognizing the unceded ancestral Indigenous lands on which a research study took place) are mentioned by Trisos et al. as a relatively easy first step towards recognizing historical inequities. Cronin et al. include a land acknowledgement, as have several other Nature Ecology \& Evolution authors to date. We encourage the inclusion of such acknowledgements where appropriate, and are working with other Nature Portfolio journals to develop standards for formatting such statements.

Two other articles published in the past year that seek to increase diversity, in very different ways, are a Correspondence from Nuñez and Amano that calls for the inclusion of non-English research in systematic reviews, and a Comment by Demery and Pipkin that presents strategies to make fieldwork safer for researchers from diverse backgrounds.

In the editorials from last year, we said that we would use our Q\&A section to showcase a more diverse range of voices, and we are proud to have published three insightful Q\&A articles in 2021: with Swanne Gordon, Kimberleigh Tommy and Jennifer Grenz. The dedicated anti-racist action channel on our community site, on the other hand, has not got off the ground. We also pledged to devote our outreach to under-represented researchers and institutions, and to decline involvement in all-white panel discussions. Unfortunately, in part owing to the pandemic, we have not been able to carry out any institutional visits this year, either virtually or in person, but we still intend to target these towards lower-income countries and minority-serving institutions in higher-income countries. We have had robust conversations about diversity with the organizers of a few panel discussions, which has resulted in some positive changes but also on one occasion in us not participating but the panel still going ahead. We need to continue to push on this issue, but possibly also to refine our approach to ensure that all outcomes are positive, rather than merely sticking to the letter of our commitment.

We continue to advocate for more inclusive practices and policies at Springer Nature. The company is considering expanding the global locations in which editorial teams can be based, is developing a new diversity, equity and inclusion strategy, and is discussing policies around parachute research. Unfortunately, we are still not in a position to systematically and appropriately collect data on the race or ethnicity of our authors and reviewers, which limits our ability to take a quantitative approach to improving and reporting on representation. However, we continue to try to broaden our reviewer pool, and have begun asking submitting authors to consider representation on their authorship panels.

As we said last year, and as many of the authors mentioned here also acknowledge, this is a long journey. But we remain committed to it and feel we have made meaningful, if still imperfect, progress so far.

Published online: 2 September 2021 https://doi.org/10.1038/s41559-021-01549-2 